\title{
A visual step-by-step guide for clinicians to use video consultations in mental health services: NHS examples of real-time practice in times of normal and pandemic healthcare delivery
}

\author{
Gemma Johns, ${ }^{1}$ [ Jacinta Tan, ${ }^{1}$ Anna Burhouse, ${ }^{2}$ Mike Ogonovsky, ${ }_{1}$ Catrin Rees, ${ }^{3}$ Alka Ahuja
}

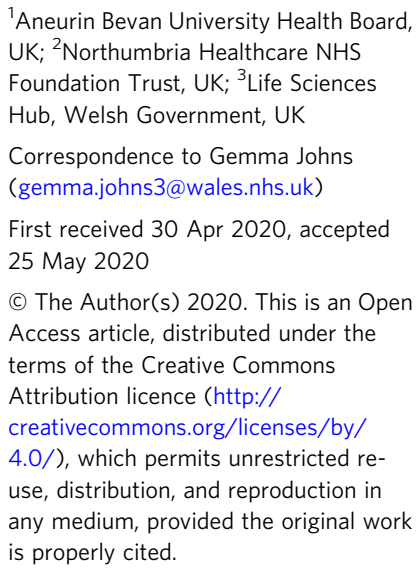

Despite the increasingly widespread use of video consultations, there are very few documented descriptions of how to set up and implement video consultations in real-time practice. This step-by-step guide will describe the set-up process based on the authors' experience of two real-time National Health Service (NHS) examples: a single health board use (delivered in normal time), and an All-Wales National Video Consultation Service roll-out (delivered during an emergency pandemic as part of the COVID-19 response). This paper provides a simple visual step-by-step guide for using telepsychiatry via the remote use of video consultations in mental health services, and outlines the mandatory steps to achieving a safe, successful and sustainable use of video consultations in the NHS by ensuring that video consultations fit into existing and new NHS workflow systems and adhere to legal and ethical guidelines.

Keywords Video consultations; COVID-19; telepsychiatry; mental health; digital health.
There is a large and growing evidence base of published data that demonstrates an overall consensus of suitability, acceptability and satisfaction regarding the use of digital technology, ${ }^{1-6}$ particularly video consultations for the purpose of remote assessments and appointments in mental health, known as 'telepsychiatry'. ${ }^{75}$ Telepsychiatry is widely reported to be at least as efficient and effective as traditional face-to-face care, providing improved clinical and quality of life outcomes across a wide range of population groups and settings. ${ }^{7-20}$ Studies have compared video consultations with standard in-person care and concluded that video consultations might be superior to in-person consultations for some forms of treatment and population groups. ${ }^{2,7}$ The evidence base for telepsychiatry remains strong and consistent across mental health studies for both adult and child services. $^{7-20}$ It is commonly argued that mental health and psychiatry are particularly well suited to video consultations and that the psychiatric interaction translates exceptionally well to the technological world. This is because many treatments are based on interpersonal 'talking therapies' and medication management, which typically do not require any other medical devices for clinical use, perhaps in contrast to other specialties. ${ }^{21}$ Video consultations have the potential to offer many additional benefits to patients, families and clinicians besides treatment. They are reported to improve and widen patient and family access to healthcare, support co-production and self-management, increase efficiency and improve clinical outcomes, as well as significantly reducing clinical time and patient and family travel. ${ }^{2,22,23}$

\section{Local evidence to support this step-by-step guide} The CWTCH quality improvement project

In 2019, the Health Foundation ${ }^{24}$ funded Aneurin Bevan University Health Board (ABUHB) for 1 year to establish a telepsychiatry programme with the objective of providing mental health appointments to children and adolescents within Gwent mental health services. This ABUHB programme is called Connecting with Telehealth to Children in Hospital and Healthcare (CWTCH). It is a National Health Service (NHS) quality improvement project that provides faster and more efficient appointments in child and adolescent mental health services (CAMHS) using a communication platform called Attend Anywhere (https://www.attendanywhere. com). The programme tested the suitability and acceptability of telepsychiatry and measured satisfaction across a wide range of settings and uses, including paediatric wards for emergency assessments, out-patient appointments, medication reviews, autism assessments, school postvention clinics for pupil suicides, virtual groups and more. It demonstrated that telepsychiatry in CAMHS is a highly suitable adjunct to routine ways of working; once people became familiar with 
this way of holding appointments, it was rated as acceptable and satisfactory by patients, families and clinicians. ${ }^{25,26}$

\section{Rebranding of CWTCH and Royal College of Psychiatry endorsement}

$\mathrm{CWTCH}$ has now been rebranded and is called $\mathrm{CWTCH}$ Cymru. It has received local and national recognition for its success and is now considered an exemplar of good practice across Wales. CWTCH Cymru and its guiding principles $^{26}$ have also been endorsed by the Welsh Royal College of Psychiatrists. ${ }^{27}$

\section{Partnership and development of the national roll-out of video consultations}

In March 2020, in response to the COVID-19 emergency, CWTCH Cymru went into partnership with Technology Enabled Care (TEC; https://digitalhealth.wales/tec-cymru) and the Welsh Government to form a National Video Consultation Service. ${ }^{28}$ This service is currently rapidly scaling up the routine use of video consultations across Wales, using the Attend Anywhere communication platform, to all appropriate primary, secondary and community care services, including mental healthcare for all ages. The National Video Consultation Service has a fully resourced website, with helpful guides, videos and toolkits, which can be accessed and used in addition to this paper. ${ }^{28,29}$

\section{Video consultation experience and lessons learned}

The experience gained from working on two very different sized projects (small versus large scale) and in two very different contexts (normal versus pandemic) has demonstrated that regardless of the scale and rate of adoption, there are distinct challenges in introducing video consultations as a new way of working in the NHS for clinicians. Clinician acceptance and use of video as an accepted alternative to established ways of working were found to be rate-limiting factors with respect to adoption and spread. This challenge is defined by the authors as 'clinician need versus clinical need'. This definition suggests that video consultations are more likely to be adopted and accepted as a feasible approach to healthcare delivery when the 'need' for this way of working is defined and accepted by the clinician or service, compared with when they are defined and accepted as a clinical need. In other words, video consultations are generally more successful and sustainable when a clinician or service perceives the need themselves and requests the service, rather when they are motivated by the projection of need or want from their patient population. It is therefore essential to establish and define this 'need' within a service, seeing clinician/service 'pull' for video consultation as an essential criterion for successful adoption. When the perceived need for video consultations by clinicians and services increased in the pandemic context, there was greater willingness to test new ways of working and overcome perceived or internal barriers to change. This has significant implications for how video consultations should be introduced into services and organisations, highlighting the need to focus on both the technical and the cultural aspects of the adoption process. These findings mirror the work of Greenhalgh et al and their 'NASSS' (non-adoption, abandonment and barriers to spread, scale-up and sustainability) framework. ${ }^{30}$

\section{Rationale and objectives}

Despite the increasingly widespread use of video consultations, there are very few visual and fully documented descriptions of how to set up and implement such consultations in real-time practice with specific attention to features such as fitting into existing systems and the legal and ethical requirements of video consultations. This step-by-step guide will describe the process based on two examples: a single health board project (delivered in normal time), and an All-Wales National Video Consultation service roll-out (delivered during the COVID-19 pandemic emergency).

\section{Methods}

This paper was designed to be a simple and visual step-by-step guide describing how to set up and use video consultations for mental health services, including a range of 'technical' skills and knowledge that clinicians and services may find helpful for the adoption of video consultations. This paper will describe the various steps that have been taken by the authors in their real-time experience to initiate and implement a new video consultation programme in an existing NHS systems. The visuals used on the TEC website and in this paper are adaptations and syntheses of work from other recent video consultation reports in the UK. ${ }^{31-33}$ Note that the visual diagrams and infographics used in this paper are examples based on the communication platform Attend Anywhere (https://www.attendanywhere.com); therefore, descriptions and instructions may differ slightly depending on the type of platform used in your health service.

Ethical approval was obtained from all seven Welsh research and development departments. Consent was obtained from all participants in both CWTCH and the National Video Consulting Service.

\section{Results}

\section{Step-by-step guide}

This section of the paper will provide a step-by-step guide that is divided up into three distinct sections. The first section demonstrates how to set up video consultations in an existing healthcare workflow. The second section provides the appropriate steps regarding ethical and legal principles. The third section discusses how to use video consultations in a secondary care health service, with the help of an infographic diagram.

\section{A guide to setting up video consultations in existing systems}

\section{Managing the video consultation service}

Within your healthcare service, you will need to identify a lead clinician who will decide how best to set up, use and manage 


\section{Work Flow: Video Consultations (VC)}
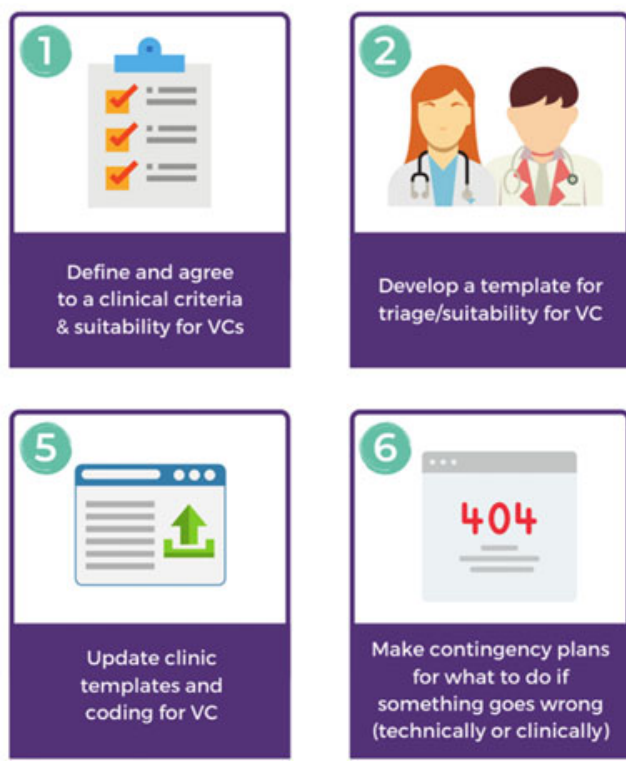
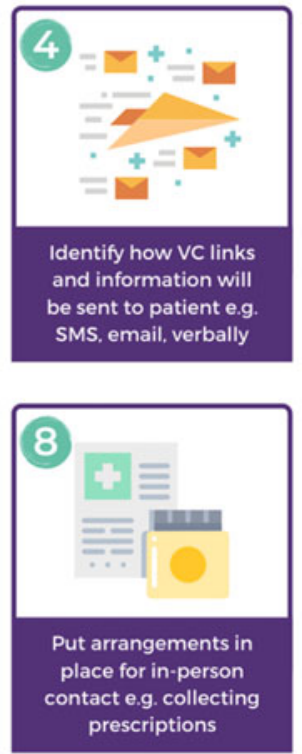

Fig. 1 Workflow visual guide. Diagram from the Welsh National Video Consultation Service Toolkit. ${ }^{29}$

the video consultations (Fig. 1). This person will be the 'point of call' for your service and its use of video consultations.

\section{Setting clinical criteria}

Your healthcare service will need to define and agree on clinical criteria for video consultations and the suitability and appropriateness of appointment types. Following this agreement, a template for triage or suitability would need to be developed and provided to the person(s) in charge of making video appointments, e.g. a receptionist.

\section{Making and managing appointments:}

Your service will need to identify how video consultations will be made, and who will make and deliver these appointments. Your service will also need to identify how appointment links and information will be sent to patients - for example, via a letter, verbally or via an SMS text or email - and how appointment slots will be offered, documented and given to the delivering clinician. In addition, your service will need to decide how the video consultation will be managed, how clinical templates and coding will be used for video consultations, and how these will be matched to existing systems. Your service will need to have a contingency plan for possible scenarios or problems (for both technical and clinical possibilities). Finally, your service will need to consider how clinical information is later documented, for example, similarly to the hand-written notes used in usual practice.

\section{Setting up the clinical space}

Your health service will need to identify how video consultations will be set up. For example, you will need to think about factors such as the room layout, e.g. whether it is welllit and well-positioned, confidentiality issues and clinical appropriateness. More about this can be found in the following sections.

\section{Additional considerations}

Your service will need to decide how to deal with providing additional information; for instance, if the picture definition obtained via a video consultation is not good enough to allow accurate visualisation and identification of skin lesions, your service needs to decide how to obtain this additional information. It is important that patients are able to receive patient information leaflets as they would in a standard consultation. Your service needs to decide the best mechanism for communicating this information to them. For example, you could email them. Your service would also need to consider additional plans for in-person contact, such as for collection of prescriptions.

\section{A guide to risk, safeguarding and ethical guidelines}

Key considerations in the use of video consultations include legal and ethical issues, such as defining and documenting patient suitability and the role of the clinician, risk assessments and contingency planning, privacy, confidentiality, security and consent (Fig. 2). Appropriate strategies covering ethical issues will be necessary, such as taking informed consent and ensuring confidentiality and security while using technology, and procedures for conducting risk assessments. 


\section{Legal \& Ethical Considerations}
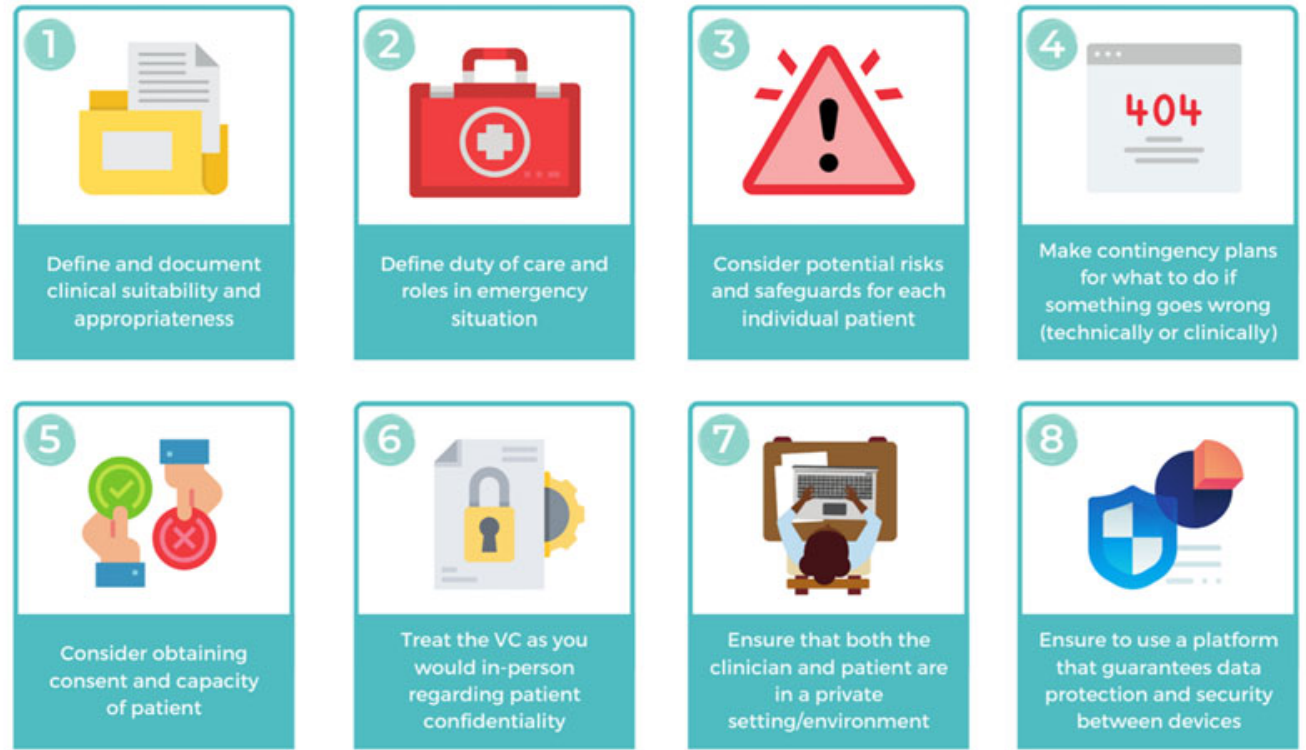

Fig. 2 Legal and ethical visual guide. Diagram from the Welsh National Video Consultation Service Toolkit. ${ }^{29}$

VC, video consultation.

\section{Define and document clinical suitability and appropriateness}

Your healthcare service will need to define and agree to clinical criteria for video consultations and the suitability and appropriateness of appointment types. This defining and documentation process would need to include the roles and responsibilities of clinical staff.

\section{Risk, safeguarding and ethical considerations}

All types of mental health services using video consultations, regardless of their level of risk, should consider conducting risk assessments, and abide by safeguarding principles and ethical guidelines.

To start thinking about the population your service will deliver a video consultation to, and the risk exposure that may arise, it is advised to conduct an initial 'process mapping' of your service. Process mapping will help your team to define video consultations in the context of your service, understand who is responsible for what and where, and to flag up any potential concerns or risk exposures. Process mapping and risk assessment will help your service to start thinking about and understanding what types of risks may arise in specific situations, and possibly how to avoid them.

You would need to think about the following.

- Would using video consultations instead of in-person care increase risk in any way?

- If so, what are these risks, and can they be resolved?

- Is the risk of using video consultations greater than not seeing the patient at all?

- Would these risks be the same if the service was delivered in person?
- What other types of risks might there be - such as the setting, environment and clinical outcome?

On completion of process mapping and initial risk assessments, the next step would be to start thinking about - and formally agreeing to and documenting clear and concise safeguarding contingency plans for your video consultation service. This would involve a 'what to do' plan in the event of an emergency or concern arising during a virtual appointment. It is advised to list a wide range of scenarios, ranging from low- to high-risk possibilities. Make it as specific to your service as possible, to make potential scenarios relatable to your staff. When developing the contingency plan, think about who is best suited to develop it and who will be following it, and consider a wide range of opinions and possibilities. Develop a list of all possible scenarios and all levels of risk exposure, and make them specific, applicable and relatable to your service.

\section{Ethical guidelines}

Video consultations, like any other form of healthcare delivery, will need to be treated exactly the same way as in-person care with regards to ethical guidelines and procedures. However, owing to the obvious remoteness of a virtual appointment, there are additional ethical considerations which need to be considered and applied, such as confidentiality, privacy and security issues.

It is essential that a video consultation service replicates an in-person appointment or assessment as much as possible. For example, the setting of an appointment room would ideally need to be the same as an in-person appointment room, e.g. if your service would normally use a private room for an in-person appointment, then a 


\section{Secondary Care Video Consultation (VC) Process}
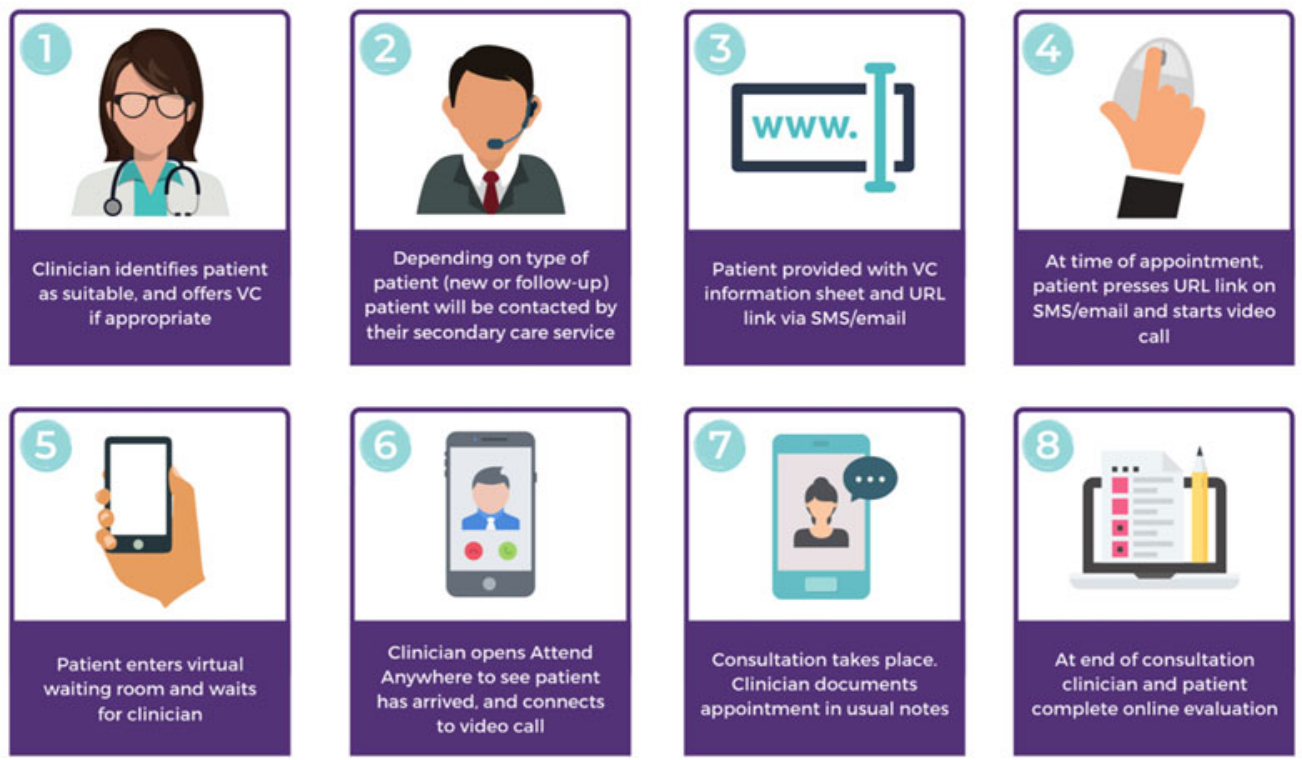

Fig. 3 How to use video consultations visual guide. Diagram from the Welsh National Video Consultation Service Toolkit. ${ }^{29}$

virtual appointment would also need this. It is also important to ensure that the platform used for video consultations is safe and secure, and that it meets your existing health systems standards, including software encryption. Many popular video chat platforms such as FaceTime and WhatsApp are not compliant with healthcare standards; therefore, you would need to seek out a safe and secure platform such as Attend Anywhere (https://www.attendanywhere.com).

Informed consent is the process of seeking agreement from a person before taking a course of action that requires consent. Informed consent is required from any person who is receiving a video consultation. There are two types of consent.

- Implied consent (or tacit consent), which is signalled by the behaviour of an informed person in agreement. This type of consent is typically used in the delivery of 'in-person' healthcare.

- Explicit consent is when a person actively agrees, either verbally or in writing. This type of consent is highly recommended for video consultations, as signalled (implied) behaviour may be more difficult to capture remotely.

To obtain consent, the person giving it would need to be considered to fully understand the process and to have full capacity to do so. A person with incapacity, such as a child or vulnerable adult, may not be able to give informed consent; therefore, parental or guardian consent (known as assent) would be required.

\section{A guide to conducting the video consultation}

The final step of this guide describes how to use video consultations in a secondary healthcare service (Fig. 3).

\section{Define and document clinical suitability and appropriateness}

As shown in the above Figs 1 and 2, your healthcare service will need to define and agree to clinical criteria and patient suitability for video consultations. This is considered the most important step for video consultations.

\section{Contact the patient and send instructions and video call link}

Depending on the type of communication platform (e.g. Attend Anywhere) you will be using, the patient will need to be offered the video appointment; they will then need to agree to it, after which they will need to be sent the patient information sheet and URL link to access the video call.

\section{Set up video call and start consultation}

Again, depending on the type of communication platform you will be using, there will need to be clear steps set out to determine how best to use video consultations in your service.

\section{Evaluation component}

To ensure that the use of video consultations is properly integrated into your health service, it is important to capture feedback on use, acceptability, suitability and satisfaction. It is therefore advisable to establish an evaluation framework to capture this. An example of this may include attaching a basic satisfaction survey to the end of the video consultation, asking a few 'how did it go' questions. 


\section{How to Use Video Consultations: Secondary Care Services}

\begin{tabular}{c|l}
1 & $\begin{array}{l}\text { Have the VC toolkit on hand: } \\
\text { digitalhealth.wales/tec-cymru }\end{array}$ \\
$\begin{array}{l}\text { Set Up } \\
\text { Prepare } \\
\text { yourself }\end{array}$ & $\begin{array}{l}\text { Watch the VC videos: } \\
\text { bit.ly/wales-vc-service }\end{array}$
\end{tabular}
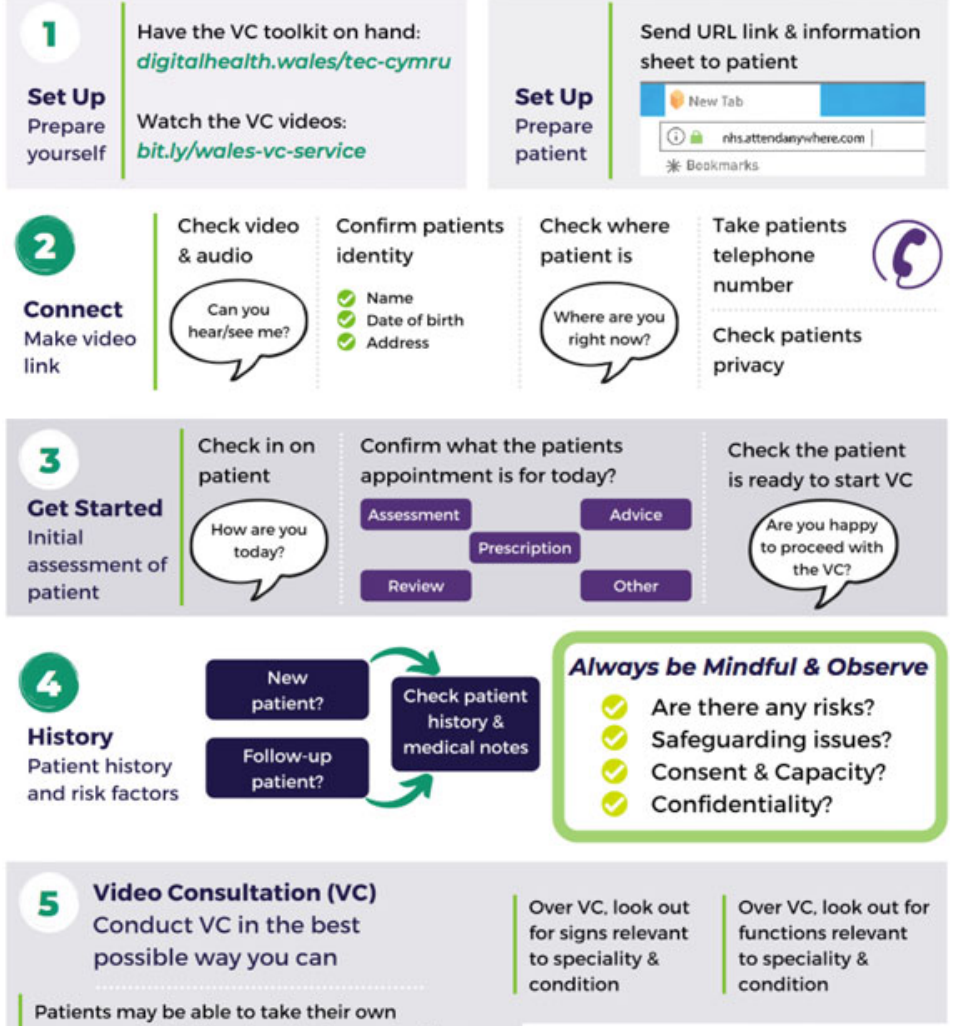

measurements if they have instruments at home
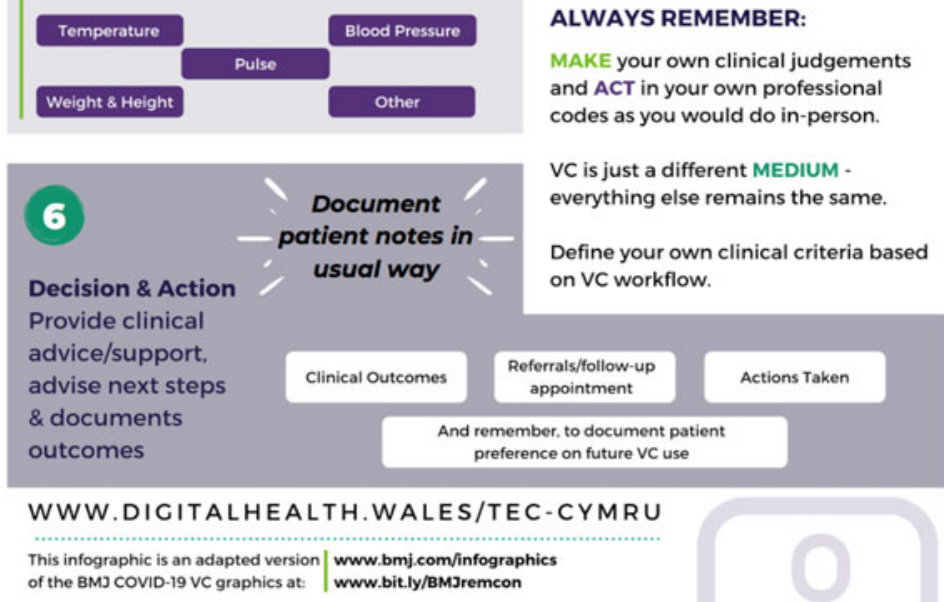

\section{ALWAYS REMEMBER:}

MAKE your own clinical judgements and $A C T$ in your own professional codes as you would do in-person. codes as you would do in-perso everything else remains the same.

Define your own clinical criteria based on VC workflow.

Provide clinical advice/support advise next steps WWW.DIGITALHEALTH.WALES/TEC-CYMRU of the BMJ COVID-19 VC graphics at: www.bit.ly/BMJremcon

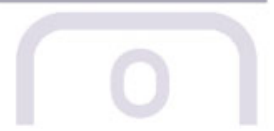

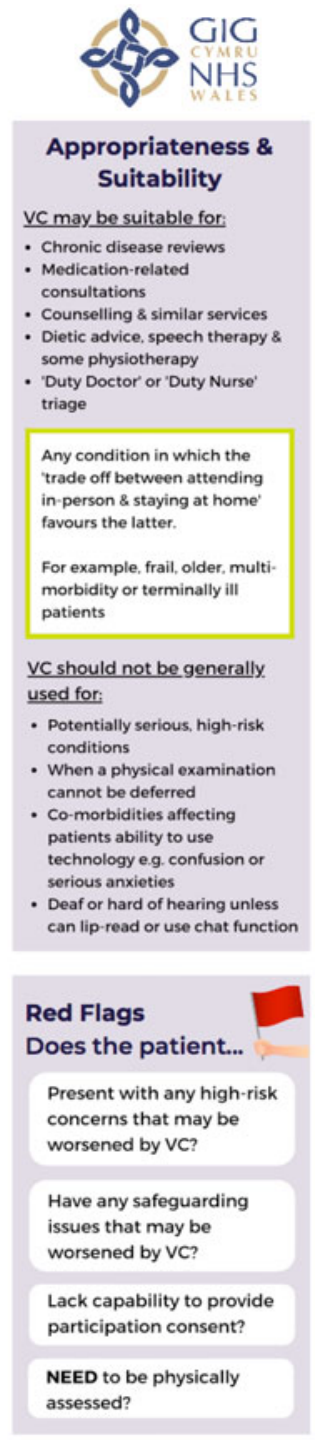

VC Workflow
Develop:
- vC clinical triage
- VC codes \& templates
- VC contingency plans
- In-person arrangements
(e.g. prescriptions)
Identifys
- How VCs will be booked
- How VCs will be
documented
- How patient links will be
sent

Fig. 4 Infographic visual guide: video consultations in secondary care. Diagram from the Welsh National Video Consultation Service Toolkit. ${ }^{29}$

For additional information, please see the TEC website (https://digitalhealth.wales/tec-cymru), which provides a detailed step-by-step guide on setting up a video consultation (Fig. 4), healthcare-specific toolkits, including examples of information sheets, suggested scripting for clinicians, technical guides to the Attend Anywhere communication platform and much more.

\section{Conclusions}

This paper outlines a simple visual step-by-step guide to help clinicians to set up and use video consultations in mental health services. This resource has been used to support clinicians to gain the technical skills and knowledge required to routinely use video consultations in practice. In addition, 
we found that there is an important 'cultural' aspect to successful adoption of video consultations, where the ratelimiting factor for successful adoption is the 'need' and 'pull' for this way of working to be defined and accepted by the clinician or ser vice.

When the perceived need for video consultations by clinicians and services increased in the pandemic context, there was greater willingness to test new ways of working and overcome perceived or internal barriers to change. It will be interesting to see whether having made the change to this new way working in the pandemic context, clinicians and services actively choose to maintain these newly gained technical skills - and also whether patients and carers, having experienced video consultations for the first time, increase their demand for this to become a new 'routine' way of working. As use of video consultations increases, we will also undoubtedly learn how to titrate this offer according to need, circumstance and demographics, and discover which healthcare treatments can best be deployed or augmented through the use of video consultations.

This paper provides a guide to using video consultations in the NHS, based on personal experience of the authors and feedback from their evaluation. However, it is still early days for video consultations in Wales, and more research is needed to understand more about their use, particularly what can and can't be done using video consultation, as this is still unspeculative and unproven.

\section{About the authors}

Gemma Johns is Research \& Evaluation Lead of CWTCH and TEC Cymru ABUHB, Wales, UK. Gemma worked on both the CWTCH and National Video Consultation project and led the evaluation for both. Jacinta Tan is a Child and Adolescent Mental Health Consultant Psychiatrist and Project Manager of CWTCH, ABUHB. Anna Burhouse is the Health Foundation QI Adviser for CWTCH and supported the evaluation of the National VC programme, Northumbria Healthcare NHS Foundation Trust. Mike Ogonovsky is Assistant Director of Informatics ABUHB and Senior Responsible Officer of TEC Cymru. Catrin Rees is at Life Sciences Hub, Welsh Government, and is a Graphics \& Web Designer for TEC Cymru. Alka Ahuja is a Child and Adolescent Mental Health Consultant Psychiatrist, Clinical Lead for TEC Cymru, Chief Investigator for CWTCH.

\section{Author contributions}

G.J. contributed to the main write up and development of the paper; she developed the manuscript draft, designed the template of the visual and infographics, and was involved in the overall structure and design, and in making amendments to the final manuscript. J.T. helped structure the manuscript and contributed clinical understanding to the guidelines, visuals and infographics. A.B. contributed some of the written text in the paper and wrote up the conclusion, and also helped structure the final manuscript and helped with amendments. M.O. contributed to the development, structure and reading of the manuscript (draft and final), and provided a technical and overall National Programme perspective on the paper and its guidelines. C.R. redesigned the draft templates into the final graphics for this paper, including the four visuals and the infographic, and also contributed to the structure and reading of the manuscript. A.A. was responsible for overseeing the entire development of the paper, helped structure the manuscript (draft and final), developed and shaped the clinical and technical perspective on the clinical guidelines provided in the paper, and helped develop the visuals and infographic.

\section{Funding}

The CWTCH project was funded by the Health Foundation as a quality improvement project in Aneurin Bevan University Health Board in Wales. The National Video Consultation Programme is currently being funded by the Welsh Government to test video consultation across all of Wales.

\section{Declaration of interest}

None

ICMJE forms are in the supplementary material, available online at https:// doi.org/10.1192/bjb.2020.71.

\section{References}

1 Kruse C, Krowski N, Rodriguez B, Tran L, Vela J, Brooks M. Telehealth and patient satisfaction: a systematic review and narrative analysis. $B M$ Open 2017; 7

2 Wootton R. Realtime telemedicine. J Telemed Telecare 2006; 12(7): 328-36.

3 Urness D, Wass M, Gordon A, Tian E, Bulger T. Client acceptability and quality of life - telepsychiatry compared to in-person consultation. Telemed Telecare 2006; 12(5): 251-4.

4 Hailey D, Roine R, Ohinmaa A. The effectiveness of tele-mental health applications: a review. Can J Psychiatry 2008; 53(11): 769-78.

5 Myers K, Vander Stoep A, Zhou C, McCarty CA, Katon W. Effectiveness of a telehealth service delivery model for treating attention-deficit/hyperactivity disorder: a community-based randomized controlled trial. J Am Acad Child Adolesc Psychiatry 2015; 54: 263-74.

6 Hilty DM, Ferrer DC, Parish MB. The effectiveness of telemental health a 2013 review. Telemed J E Health 2013; 19: 444-54.

7 Bashshur RL, Shannon GW, Bashshur N, Yellowlees PM. The empirical evidence for telemedicine interventions in mental disorders. Telemed J $E$ Health 2016; 22(2): 87-113.

8 Hilty DM, Nesbitt TS, Kuenneth CA, Cruz GM, Hales RE. Rural versus suburban primary care needs, utilization, and satisfaction with telepsychiatric consultation. J Rural Health 2007; 23(2): 163-5.

9 Hilty DM, Nesbit TS, Marks SL, Callanhanm EJ. Effects of telepsychiatry on the doctor-patient relationship: communication, satisfaction, \& relevant Issues. Prim Psychiatry 2002; 9: 29-34.

10 Myers KM, Valentine JM, Melzer SM. Child and adolescent telepsychiatry utilisation and satisfaction. Telemed J E Health 2008; 14: 131-7.

11 García-Lizana F, Muñoz-Mayorga I. What about telepsychiatry? A systematic review. Prim Care Companion J Clin Psychiatry 2010; 12(2): https://doi.org/10.4088/pcc.09m00831whi.

12 Neufeld JD, Yellowlees PM, Hilty DM, Cobb H, Bourgeois JA. The eMental Health Consultation Service: providing enhanced primary-care mental health services through telemedicine. Psychosomatics 2007; 48(2): 135-41.

13 Hubley S, Lynch SB, Schneck C, Thomas M, Shore J. Review of key telepsychiatry outcomes. World J Psychiatry 2016; 22(6): 269-82.

14 Diamond JM, Bloch RM. Telepsychiatry assessments of child or adolescent behaviour disorders: a review of evidence and issues. Telemed J E Health 2010; 16: 712-6.

15 Myers KM, Sulzbacher S, Melzer S. Telepsychiatry in child and adolescent psychiatry. Are patients comparable to those in usual outpatient care? Telemed J Health 2004; 10: 248-84.

16 Elford R, White H, Bowering R, Ghandi A, Maddiggan B, St John K., et al. $A$ randomised controlled trial of child psychiatric assessments conducted using videoconferencing. J Telecare 2000; 6: 73-82.

17 Yellowlees PM, Hilty DM, Marks SL, Neufeld J, Bourgeouis JA. A retrospective analysis of child and adolescent eMental Health program. J Am Acad Child Adolescent Psychiatry 2008; 47: 103-7.

18 Hyler SE, Gangure DP, Batchelder ST. Can telepsychiatry replace in-person psychiatric assessments? A review and meta-analysis of comparison studies. CNS Spectrums 2005; 10(5): 403-13. 
19 Himle MB, Freitag M, Walther M, Franklin SA, Ely L, Woods DW. A randomized pilot trial comparing videoconference versus face-to-face delivery of behaviour therapy for childhood tic disorders. Behav Res Ther 2012; 50: 565-57.

20 Kruse CS, Krowski N, Rodriguez B, Tran L, Vela J, Brooks M. Telehealth and patient satisfaction: a systematic review and narrative analysis. BMJ Open 2017; 7: e016242.

21 Spaulding R, Cain S, Sonnenschein K. Urban telepsychiatry: uncommon service for a common need. Child Adolesc Psychiatr Clin North Am 2011; 20: 29-39

22 Rabinowitz T, Murphy KM, Amour JL, Ricci MA, Caputo MP, Newhouse $P A$. Benefits of a telepsychiatry consultation service for rural nursing home residents. Telemed J E Health 2010; 16(1): 34-40.

23 E-Health Scotland. New Mental Health Strategy. E-Health Scotland, 2017 (https://www.ehealth.scot/scotlands-new-mental-health-strategy/).

24 The Health Foundation. Introducing Telepsychiatry in Child and Adolescent Mental Health Services. The Health Foundation, 2019 (https://www. health.org.uk/improvement-project/introducing-telepsychiatry-inchild-and-adolescent-mental-health-services).

25 Technology Enabled Care Cymru. News \& Blogs, from the CWTCH Cymru Project. Digital Health Wales, 2020 (https://digitalhealth.wales/news?).

26 Tan J, Johns G, Ahuja A. The CWTCH Cymru Toolkit, Ready, Set, Go! Royal College of Psychiatrists in Wales, CWTCH, 2020 (https:// www.rcpsych.ac.uk/docs/default-source/members/divisions/wales/ cwtch-ready-set-go-toolkit.pdf).
27 Royal College of Psychiatrists. CWTCH: Connecting with Telehealth to Communities and Hospitals in Healthcare. RCPsych, 2020 (https://www. rcpsych.ac.uk/members/devolved-nations/rcpsych-in-wales/news/connecting-with-telehealth-to-communities-and-hospitals-for-healthcare).

28 TEC Cymru. NHS Wales Video Consulting Service. TEC Cymru, no date (https://digitalhealth.wales/tec-cymru/vc-service).

29 TEC Cymru. Video Consulting Toolkits. TEC Cymru, no date (https:// digitalhealth.wales/tec-cymru/nhs-wales-video-consulting-service/ video-consulting-toolkits)

30 Greenhalgh T, Wherton J, Papoutsi C, Lynch J, Hughes G, A'Court C et al. Beyond adoption: a new framework for theorizing and evaluating nonadoption, abandonment, and challenges to the scale-up, spread, and sustainability of health and care technologies. J Med Internet Res 2017; 19(11): e367.

31 Nuffield Department of Primary Care Health Sciences, Medical Sciences Division. Video Consulting in the NHS. Nuffield Department of Primary Care Health Sciences, Medical Sciences Division, no date (https:// www.phc.ox.ac.uk/research/resources/video-consulting-in-the-nhs).

32 BJGP Life. Video Consultations: A Guide in Practice. BJGP Life, 18 Mar 2020 (https://bjgplife.com/2020/03/18/video-consultations-guide-for-practice/).

33 Greenhalgh T, Koh GCH, Car J. COVID-19: a remote assessment in primary care. BMJ 2020; 368: $\mathrm{m} 1182$.

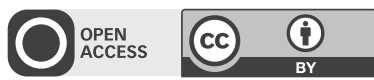

\title{
INTERVIEW
}

\section{Kenneth Kendler: fully human}

\author{
Claire McKenna $\odot$ talks to Kenneth Kendler, psychiatric polymath, about the \\ consolations of philosophy, poetry and theology
}

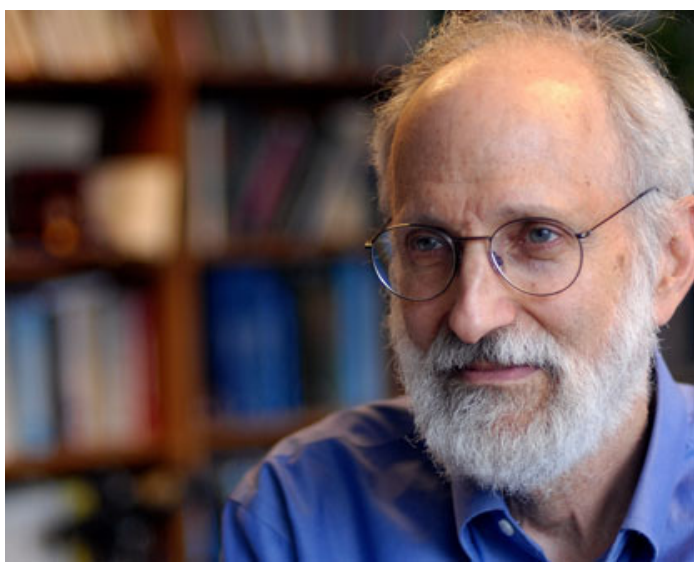

'We shall not cease from exploration

And the end of all our exploring

Will be to arrive where we started

And know the place for the first time.'

T. S. Eliot, 'Little Gidding' (from Four Quartets), 1942

Born the son of two experimental psychologists (his father a 'classic New York Jewish character', his mother a 'very intellectual, incisive' woman who 'overcame a lot of prejudice about being a woman in academia'), Professor Kenneth Kendler's parents were initially sceptical about his choice of a career in psychiatry: 'In my parents' days (they would have trained in the late 1930s and early 1940s), the smart people went on to get a PhD and the physicians were sort of second class. They basically were either psychoanalysts or they went to state hospitals and gave lots of people ECT... My father, for example, worked in the mental health system during the Second World War. So he had a lot of contact with psychiatrists and was not, on average, very impressed'. He thinks he made them proud in the end though: 'I think eventually I convinced them one could do rigorous research in this area'.

At the time of writing, Kendler is the second most highly cited psychiatric researcher in the world. Already a renowned expert in psychiatric genetics, he diverged later in his career to become an eminent scholar in the philosophy, and latterly the history, of psychiatry. He has gone to the lengths of employing a German speaking translator for the past few years, so he can read Emil Kraepelin's writings in the original. These later interests were in part an attempt, it seems, to resolve his own sturm und drang about classification in psychiatry: 'You cannot understand where we are in psychiatric nosology without understanding the historical 